\title{
Regulation of the Intracellular Location of Methane Mono-oxygenase During Growth of Methylosinus trichosporium OB3b on Methanol
}

\author{
By KAREN J. DAVIS, * ALEX CORNISH† AND I. JOHN HIGGINS \\ Biotechnology Centre, Cranfield Institute of Technology, Cranfield, Bedfordshire MK43 OAL, UK
}

(Received 12 May 1986; revised 2 September 1986)

The methane-oxidizing bacterium Methylosinus trichosporium OB3b retains the capacity to express two types of methane mono-oxygenase (MMO) during growth on methanol in continuous culture. Soluble MMO predominates during copper-limited growth whilst particulate (membrane-bound) MMO activity is expressed fully only in copper-sufficient cultures. Organisms containing soluble MMO oxidized ethylbenzene (an artificial substrate for soluble but not particulate MMO) but this capacity was progressively lost when additional copper was made available to the organism, and MMO activity was transferred to the particulate fraction of cell-free extracts. Although intracellular location of MMO is apparently regulated by copper availability during growth on both methane and methanol, whole-cell MMO activities are appreciably higher during growth on the former (natural) carbon source.

\section{INTRODUCTION}

Methanotrophs oxidize methane using the sequence of enzyme-catalysed reactions shown in Fig. 1 (Higgins et al., 1981). The oxidation of methane to methanol is catalysed by methane mono-oxygenase (MMO) systems, of which two distinct types, one soluble and the other particulate (membrane-bound), occur in both Methylosinus trichosporium OB3b (Scott et al., 1981; Burrows et al., 1984) and Methylococcus capsulatus (Ribbons \& Wadzinski, 1976; Stanley et al., 1983) during growth on methane. The soluble MMO oxidizes a wide range of hydrocarbons in addition to methane (e.g. $n$-alkanes, $n$-alkenes, aromatic and alicyclic compounds) and uses only NAD(P)H as an electron donor (Colby et al., 1977; Stirling et al., 1979; Burrows et al., 1984). Particulate MMO, on the other hand, oxidizes only $n$-alkanes and $n$ alkenes (Dalton et al., 1984; Burrows et al., 1984) and functions with NAD(P)H and additional electron donors (Leek \& Dalton, 1983; Cornish et al., 1985). Copper availability determines which MMO is present in both $M$. trichosporium OB3b and Methylococcus capsulatus (Bath) during growth on methane. Particulate MMO exists when the organisms are grown under conditions of copper sufficiency, whilst soluble MMO exists when they experience copper deficiency (Stanley et al., 1983; Burrows et al., 1984; Dalton et al., 1984).

Some methanotrophs can also grow on methanol, although on this carbon source MMO is theoretically unnecessary for growth (Fig. 1). There have been conflicting reports concerning the levels of MMO activity and the intracellular location of the methane oxidizing system when methanotrophs are grown on methanol instead of methane. Hou et al. (1979) reported that several methanol-grown methanotrophs, including $M$. trichosporium OB3b, lost the capacity to oxidize methane. By contrast, Best \& Higgins (1981) found that $M$. trichosporium OB3b retained MMO activity during growth on methanol in batch culture and carbon-limited continuous culture and MMO activity was always located in the soluble fraction of cell-free extracts. Prior \& Dalton (1985) reported that Methylococcus capsulatus (Bath) contained only particulate MMO

† Present address: Department of Biochemistry, University of Leicester, Leicester LE1 7RH, UK.

Abbreviation: MMO, methane mono-oxygenase.

0001-3449 (C) 1987 SGM 


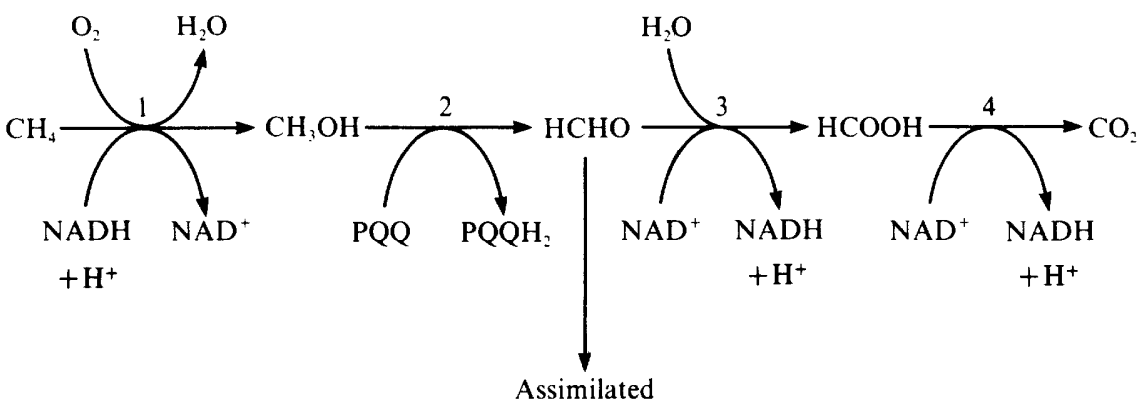

Fig. 1. Generalized scheme for the oxidation of methane by methanotrophs. 1, MMO; 2, methanol dehydrogenase; 3 , formaldehyde dehydrogenase; 4 , formate dehydrogenase; $P Q Q$, pyrrolo-quinoline quinone.

activity during growth on methanol in carbon-limited continuous culture: soluble MMO was never detected, even when the organism was grown on a copper-free medium, but particulate MMO activity increased significantly when additional copper was made available.

It has been suggested that the 'soluble' MMO activity reported by Best \& Higgins (1981) might have been due to particulate MMO released from membranes during sonic disruption of M. trichosporium OB3b (Prior \& Dalton, 1985). This seems unlikely, since the methanol-grown cultures used by Best \& Higgins (1981) had the capacity to hydroxylate ethylbenzene, which is a substrate for soluble but not particulate MMO (Burrows et al., 1984). Similar findings have been reported by Jezequel \& Higgins (1983) and Jezequel et al. (1984). These reports suggest that soluble MMO can indeed exist in methanol-grown $M$. trichosporium $\mathrm{OB} 3 \mathrm{~b}$, although particulate MMO has not been reported.

The present study investigates the effect of growth conditions, in particular copper availability, on the intracellular location of MMO in $M$. trichosporium OB3b during growth on methanol. Our results show that this organism contains soluble or particulate MMO (or both) during growth on this carbon source.

\section{METHODS}

Organism and growth conditions. Methylosinus trichosporium OB3b was grown on a nitrate salts medium (NSM) (Cornish $e t$ al., 1984). $\mathrm{CuSO}_{4}$ was added to a final concentration of $1 \mu \mathrm{M}$ unless otherwise stated. The components of the growth medium were dissolved in glass-distilled water. Water purified by reverse-osmosis followed by treatment with activated charcoal, resin ion exchange and ultrafiltration $(2 \mu \mathrm{m})$ (Elga) was used as indicated.

$M$. trichosporium OB3b was first grown on NSM agar with methane as the carbon source, and adapted to grow on methanol $(1 \%, \mathrm{v} / \mathrm{v})$ at $30^{\circ} \mathrm{C}$ in shake-flask culture as described previously (Best \& Higgins, 1981 ; Cornish et al., 1984) except that the NSM medium was supplemented with filter sterilised $\mathrm{NaHCO}_{3}(0.05 \%$, w/v) as this was found to assist initial growth (all cultures from 12 trials grew on methanol in the presence of $\mathrm{NaHCO}_{3}$, whereas $80 \%$ failed to grow in its absence). Growing cultures were used to inoculate the fermenter.

The organism was grown on methanol $(1 \%, v / v)$ in carbon-excess continuous culture using a 41 capacity LKB Ultroferm fermenter (temperature, $30^{\circ} \mathrm{C}$; impeller speed, 300 r.p.m.; $D=0.04 \mathrm{~h}^{-1}$ ). The growth limiting nutrient was varied as stated in the text.

Measurement of dissolved oxygen. This was done using an Orbisphere 2607 instrument fitted with a model 2111 sensor, connected to a chart recorder. The probe was sterilized by immersion in an aqueous solution of ethylene oxide $(20 \%, \mathrm{v} / \mathrm{v})$ overnight before insertion into the fermenter vessel. The instrument was calibrated in airsaturated water according to the manufacturer's instructions.

Preparation of whole-organism suspensions and cell-free extracts. These were prepared as described by Burrows et al. (1984).

Assay of MMO activity. All assays were carried out as described by Burrows et al. (1984). MMO activity of whole-organism suspensions was measured by following the epoxidation of propene ( $20 \mathrm{mM}$-sodium formate was used as a source of reducing equivalents) using gas chromatography. MMO activity in soluble and particulate fractions of cell-free extracts was also measured by following the epoxidation of propene but NADH (5 mM) was used as electron donor. 
Measurement of dry weight and protein. Suspensions of $M$. trichosporium OB3b were washed once using distilled water and dried at $100^{\circ} \mathrm{C}$ to constant weight. Protein content of cell-free preparations was measured using the modified Lowry assay of Peterson (1977). Bovine serum albumin was used as standard.

$S D S-P A G E$. Electrophoresis was carried out on $12 \%(\mathrm{w} / \mathrm{v})$ acrylamide gels using the discontinuous buffer system of Laemmli (1970). Proteins were fixed, stained with Coomassie blue and the gels destained as described by Laemmli (1970). The following proteins were used as standards $\left(M_{\mathrm{r}}\right.$ in parentheses): lactalbumin (14200); trypsin inhibitor (20100); trypsinogen (24000); carbonic anhydrase (29000); glyceraldehyde-3-phosphate dehydrogenase (36000); egg albumin (45000); bovine albumin (66000). Methanol dehydrogenase from $M$. trichosporium OB3b $\left(M_{\mathrm{r}} 60000\right)$ was used as a further standard. This was a gift from Dr D. J. Best, Cranfield Institute of Technology.

Chemicals. All chemicals were obtained from BDH except for $M_{\mathrm{r}}$ standards, which were purchased from Sigma.

\section{RESULTS}

\section{Effect of growth conditions on the intracellular location of $M M O$}

$M$. trichosporium $\mathrm{OB} 3 \mathrm{~b}$ was initially grown on methanol $(1 \%, \mathrm{v} / \mathrm{v})$ in continuous culture $(D=$

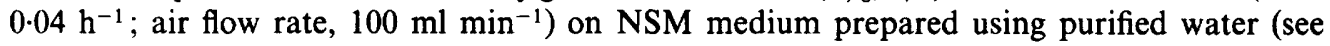
Methods). The normal concentration of $\mathrm{Cu}^{2+}$ was $1 \mu \mathrm{M}$. Under these growth conditions the culture was $\mathrm{O}_{2}$-limited and $34-57 \%$ of the total MMO activity was located in the soluble fraction of cell-free extracts (Table 1). The proportion of soluble MMO could be increased by imposing a copper limitation on the organism: this was accomplished either by omitting $\mathrm{CuSO}_{4}$ from the growth medium, in which case the biomass density of the culture decreased, or by increasing the aeration rate (and hence $\mathrm{O}_{2}$ availability), in which case the biomass density rose by a small but significant amount prior to onset of copper limitation (Table 1). For a given aeration rate, the proportion of particulate MMO increased only when the copper concentration was raised and the culture allowed to become copper-sufficient (Table 1). The same pattern of MMO regulation was observed when $M$. trichosporium OB3b was grown on NSM medium prepared using glassdistilled water, except that the biomass densities of steady state cultures were significantly lower for a given amount of added copper (Table 1). This was presumably because the copper content of the glass-distilled water was lower than that of the purified water used in previous experiments.

\section{Table 1. Effect of growth conditions on the intracellular location of MMO in methanol-grown M. trichosporium $O B 3 b$}

M. trichosporium OB3b was grown on methanol $(1 \%, \mathrm{v} / \mathrm{v})$ in continuous culture $\left(D=0.04 \mathrm{~h}^{-1}\right)$. The amount of $\mathrm{CuSO}_{4}$ added to the medium feed and the availability of $\mathrm{O}_{2}$ were varied as indicated. The growth medium was prepared using either purified water $(a)$ or glass-distilled water $(b)$ (see Methods). Most data are given as the mean \pm SEM of measurements carried out on separate days; the numbers of independent determinations are given in parentheses.

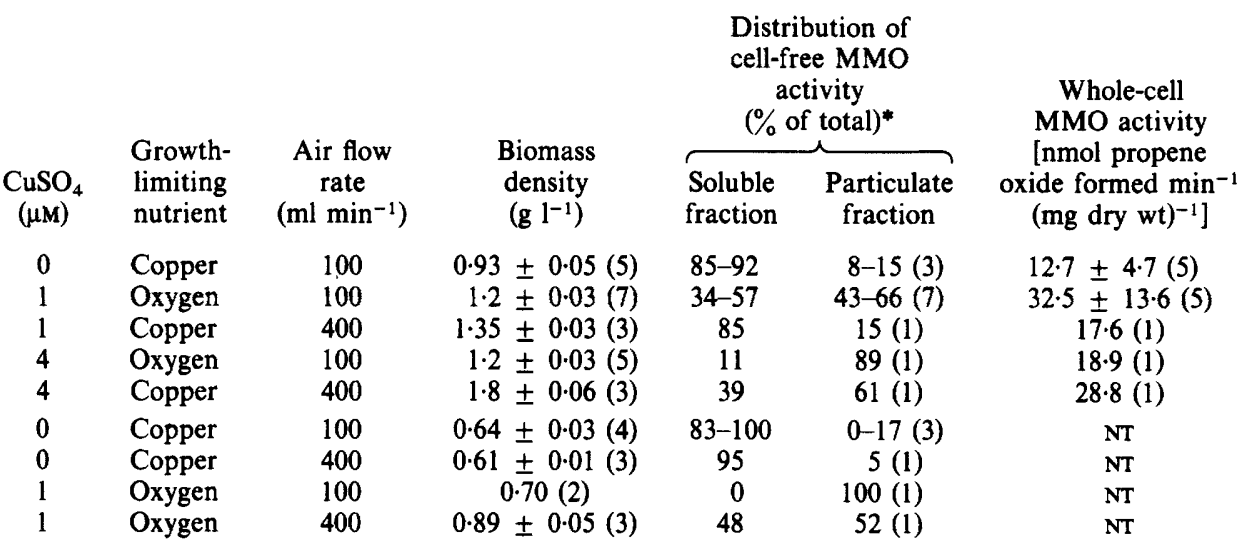

NT, Not tested.

* $100 \%$ MMO activity is defined as the activity found in the soluble fraction of cell-free extracts, measured by following propene oxidation, plus that found in the particulate fraction of the same sample. 

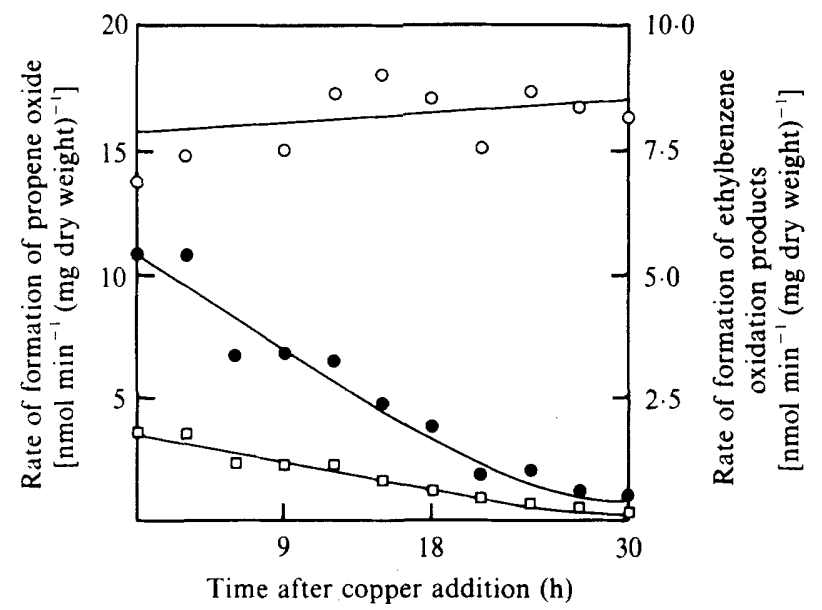

Fig. 2. Oxidation of propene and ethylbenzene by $M$. trichosporium OB3b following relief of copper deficiency. The organism was initially grown on methanol $(1 \%, v / v)$ in continuous culture under copper limitation $\left(D=0.04 \mathrm{~h}^{-1}\right.$; air flow rate, $100 \mathrm{ml} \mathrm{min}^{-1}$; biomass density, $0.9 \mathrm{~g} \mathrm{l}^{-1}$; NSM medium, nominally copper-free, prepared using purified water). Under these conditions $92 \%$ of the MMO activity was located in the soluble fraction of cell-free extracts. Particulate MMO was induced by adding $\mathrm{CuSO}_{4} \cdot 5 \mathrm{H}_{2} \mathrm{O}$ to the culture vessel to give a final concentration of $4 \mu \mathrm{M}-\mathrm{Cu}^{2+}$; the $\mathrm{Cu}^{2+}$ concentration of the medium feed was increased to $4 \mu \mathrm{M}$ at the same time. Samples were withdrawn from the chemostat at intervals following copper addition and rates of oxidation of propene and ethylbenzene by whole organisms were measured by following product formation using gas chromatography. $\bigcirc$, Propene oxide;, 1 -phenylethanol; $\square$, 4-hydroxyethylbenzene.

As expected, the ambient concentration of $\mathrm{O}_{2}$ in copper-limited cultures rose as the aeration rate was increased, whereas the $\mathrm{O}_{2}$ concentration was below the limit of detection in coppersufficient, $\mathrm{O}_{2}$-limited cultures (Table 1). Interestingly, the dissolved $\mathrm{O}_{2}$ concentration of a copper-limited culture $\left(D=0.04 \mathrm{~h}^{-1}\right.$; biomass density, $0.61 \mathrm{~g} \mathrm{l}^{-1}$; no added $\mathrm{Cu}^{2+}$; air flow rate, $100 \mathrm{ml} \mathrm{min}-1$; NSM medium prepared using glass-distilled water) decreased exponentially with time $\left(t \frac{1}{2}=45 \mathrm{~min}\right)$ from 5.4 to $1.1 \mu \mathrm{M}$ within $2 \mathrm{~h}$ of adding $\mathrm{CuSO}_{4}(1 \mu \mathrm{M})$. The kinetics of this decay suggest that during copper-limited growth the organism may contain a protein that is potentially capable of reducing molecular $\mathrm{O}_{2}$ and which is activated when additional copper is made available (see also Prior \& Dalton, 1985).

\section{MMO activity during growth on methanol}

Whole cells of $M$. trichosporium $\mathrm{OB} 3 \mathrm{~b}$ retained MMO activity during growth on methanol under all conditions tested, as judged from their capacity to oxidize propene to propene oxide in the presence of sodium formate (Table 1). The rates of propene oxide formation were, however, significantly lower than those reported for washed whole-cell suspensions of methane-grown $M$. trichosporium OB3b [i.e. $90-160 \mathrm{nmol}$ propene oxide formed $\mathrm{min}^{-1}$ (mg dry weight) ${ }^{-1}$ ] (Burrows et al., 1984; Cornish et al., 1984). Organisms that had been grown under conditions of copper deficiency and contained soluble MMO were, in addition, capable of oxidizing ethylbenzene (which is a substrate for the soluble but not particulate MMO) to 4-hydroxyethylbenzene and 1phenylethanol, but this capacity was progressively lost when additional copper was introduced to the growth medium and MMO activity transferred to the particulate fraction of cell-free extracts (Fig. 2). The rate of propene oxidation by whole-cell suspensions altered little during this time (Fig. 2). The change in intracellular location of MMO was accompanied by the disappearance of four abundant, soluble polypeptides $\left(M_{\mathrm{r}} 57000,45000,23000\right.$ and 16500$)$ and the concomitant increase in the concentration of a membrane-bound polypeptide $\left(M_{\mathrm{r}} 42000\right)$ (Fig. 3). The concentration of these polypeptides changed in essentially the same fashion following the addition of copper to cultures of $M$. trichosporium $\mathrm{OB} 3 \mathrm{~b}$ growing on methane under 


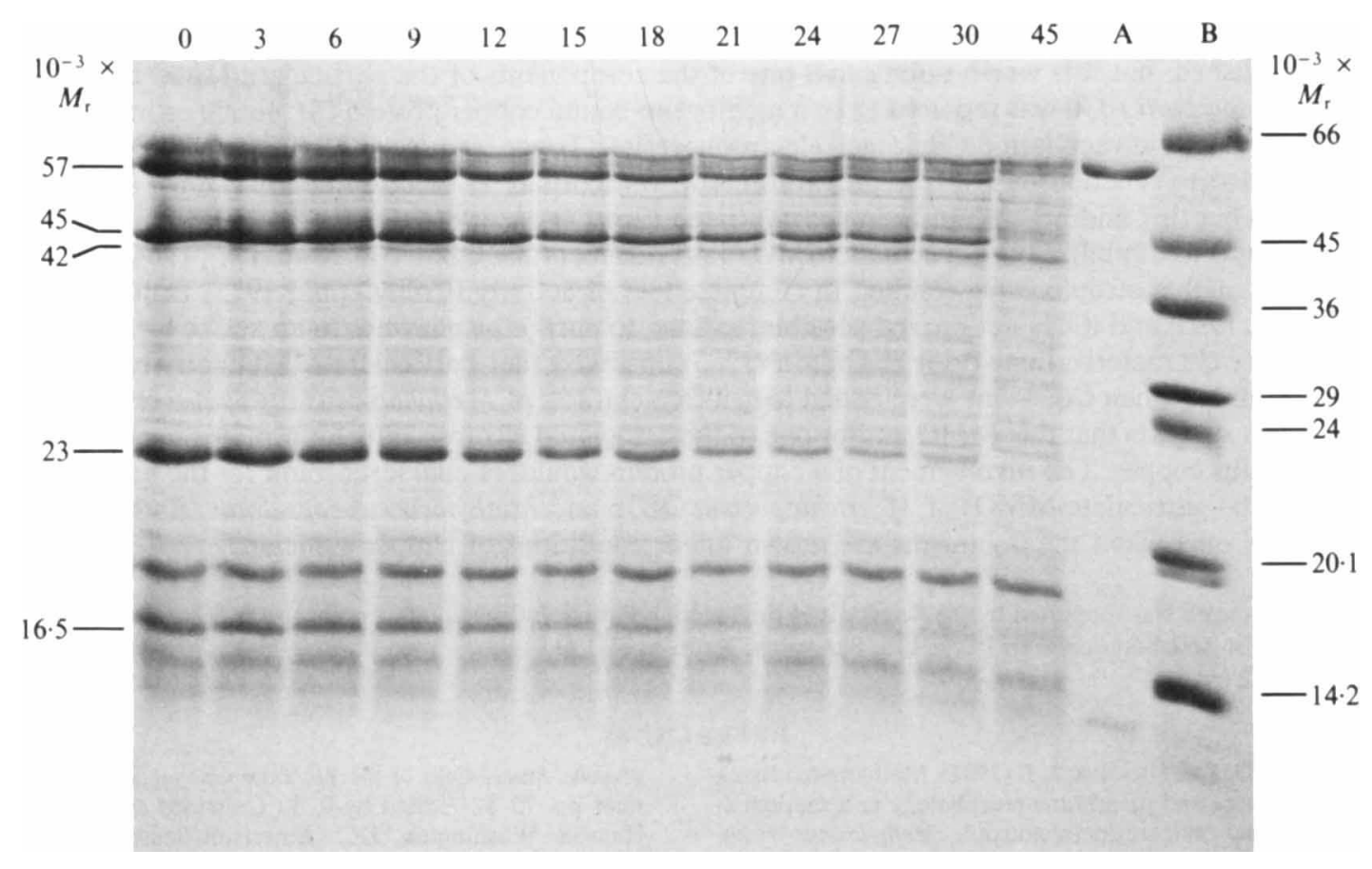

Fig. 3. Changes in the concentrations of cellular polypeptides following addition of $\mathrm{CuSO}_{4} \cdot 5 \mathrm{H}_{2} \mathrm{O}$ to a copper deficient culture of $M$. trichosporium OB3b. The figure, a photograph of an SDS-PAGE gel, shows the protein profiles of whole cells of $M$. trichosporium OB3b withdrawn from the chemostat during the experiment described in the legend to Fig. 2. Numbers above the gel tracks denote times of sampling (h) following addition of copper. Track B contained $M_{\mathrm{r}}$ standards, track A pure methanol dehydrogenase of $M$. trichosporium $\mathrm{OB} 3 \mathrm{~b}$.

conditions of copper deficiency, except that the soluble protein of $M_{\mathrm{r}} 16500$ was not noted previously (Burrows et al., 1984). The precise function of these polypeptides is at present uncertain. Three of the soluble polypeptides $\left(M_{\mathrm{r}} 57000,45000\right.$, and 23000$)$, however, are almost identical in size to the three hydroxylase subunits of soluble MMO isolated from Methylococcus capsulatus (Bath) (Woodland \& Dalton, 1984) and Methylobacterium sp. strain CRL-26 (Patel, 1984 ) and may well be constituents of the corresponding enzyme from $M$. trichosporium OB3b.

$M$. trichosporium $\mathrm{OB} 3 \mathrm{~b}$ was grown on methanol in continuous culture for up to $1600 \mathrm{~h}$ (approximately 90 generations). The organism retained the capacity to grow on methane without discernible lag after this time, indicating that the methane-oxidizing system(s) were still functional despite prolonged cultivation on an alternative carbon source.

\section{DISCUSSION}

The objective of the present work was to establish whether M. trichosporium OB3b retains the capacity to express two types of MMO (i.e. soluble and particulate) during growth on methanol. Our results show that this is indeed the case and indicate that copper availability governs which type of MMO predominates during growth of $M$. trichosporium OB3b on methanol as well as on methane (Stanley et al., 1983; Burrows et al., 1984). M. trichosporium OB3b is the only methanotroph in which both types of MMO have been found to occur during growth on methanol. Intracellular location of MMO may be regulated differently in Methylococcus capsulatus (Bath) during growth on methanol, however, since this organism expressed only particulate MMO during growth on methanol, even when copper was omitted from the growth medium (Prior \& Dalton, 1985). 
The precise mechanism(s) by which copper availability regulates MMO remains to be established, but it is worth noting that one of the components of the particulate MMO of $M$. trichosporium $\mathrm{OB} 3 \mathrm{~b}$ was reported to be a membrane-bound copper protein $\left(M_{\mathrm{r}} 46000\right.$ estimated using SDS-polyacrylamide disc gel electrophoresis) (Tonge et al., 1977). This MMO was reported to function in cell-free preparations with ascorbate as electron donor (Tonge et al., 1975) but this finding has not been substantiated (Scott et al., 1981). Furthermore, subsequent attempts to solubilize the particulate MMO of $M$. trichosporium OB3b (Burrows et al., 1984) and other methanotrophs have resulted in complete loss of activity (Colby et al., 1975; Dalton \& Leak, 1985) and it has not proved possible, to date, to purify the enzymes in an active form for further characterization. Prior \& Dalton (1985) observed that particulate MMO activity was stimulated when $\mathrm{Cu}^{2+}$ ions were added to cell-free extracts of Methylococcus capsulatus (Bath), which suggests that this MMO and/or one of the associated electron transfer proteins probably contains copper. The involvement of a copper protein would, of course, account for the finding that the particulate MMOs of $M$. trichosporium OB3b and Methylococcus capsulatus (Bath) are active only when the organisms are grown under conditions of copper sufficiency.

This work was supported by the Science and Engineering Research Council via a grant to I. J.H. and a Case award in collaboration with Pfizer Ltd, Sandwich, Kent, to K. J.D.

\section{REFERENCES}

BEST, D. J. \& HigGINS, I. J. (1981). Methane-oxidizing activity and membrane morphology in a methanolgrown obligate methanotroph, Methylosinus trichosporium OB3b. Journal of General Microbiology 125, 73-84.

Burrows, K. J., Cornish, A., Scott, D. \& Higgins, I. J. (1984). Substrate specificities of the soluble and particulate methane mono-oxygenases of Methylosinus trichosporium OB3b. Journal of General Microbiology 130, 3327-3333.

Colby, J., Dalton, H. \& WhitTenbury, R. (1975). An improved assay for bacterial methane mono-oxygenase: some properties of the enzyme from Methylomonas methanica. Biochemical Journal 151, 459-462.

Colby, J., Stirling, D. I. \& Dalton, H. (1977). The soluble methane mono-oxygenase of Methylococcus capsulatus (Bath). Its ability to oxygenate $n$-alkanes, $n$-alkenes, ethers and alicyclic, aromatic and heterocyclic compounds. Biochemical Journal 165, 395402.

Cornish, A., Nichols, K. M., Scott, D., Hunter, B. K., Aston, W. J., Higgins, I. J. \& SANDERS, J. K. M. (1984). In vivo ${ }^{13} \mathrm{C}-\mathrm{NMR}$ investigations of methanol oxidation by the obligate methanotroph, Methylosinus trichosporium OB3b. Journal of General Microbiology 130, 2565-2575.

Cornish, A., MaCDonald, J., Burrows, K. J., King, T. S., ScotT, D. \& Higgins, I. J. (1985). Succinate as an in vitro electron donor for the particulate methane mono-oxygenase of Methylosinus trichosporium OB3b. Biotechnology Letters 7, 319-324.

Dalton, H. \& LEAK, D. J. (1985). Mechanistic studies on the mode of action of methane mono-oxygenase. In Gas Enzymology, pp. 169-186. Edited by H. Degn, R. P. Cox \& H. Toftlund. Dordrecht, The Netherlands: D. Reidel Publishing Co.

Dalton, H., PrioR, S. D., Leak, D. J. \& Stanley, S. H. (1984). Regulation and control of methane mono-oxygenase. In Microbial Growth on $C_{1}$ Com- pounds. Proceedings of the 4th International Symposium, pp. 75-82. Edited by R. L. Crawford \& R. S. Hanson. Washington, DC: American Society for Microbiology.

Higgins, I. J., Best, D. J., Hammond, R. C. \& Scott, D. (1981). Methane-oxidising microorganisms. Microbiological Reviews 45, 556-590.

hou, C. T., Patel, R., Laskin, A. I. \& Barnabe, N. (1979). Microbial oxidation of gaseous hydrocarbons: epoxidation of $\mathrm{C}_{2}-\mathrm{C}_{4}$-alkanes by methylotrophic bacteria. Applied and Environmental Microbiology 38, 127-134.

Jezequel, S. G. \& Higgins, I. J. (1983). Mechanistic aspects of biotransformations by the mono-oxygenase system of Methylosinus trichosporium OB3b. Journal of Chemical and Technical Biotechnology 33B, 139-144.

Jezequel, S. G., KaY, B. \& Higgins, I. J. (1984). $O$ Dealkylation - a newly discovered class of reactions catalysed by the soluble MMO of the methanotroph Methylosinus trichosporium OB3b. Biotechnology Letters 5, 567-570.

LAEMMLI, U. K. (1970). Cleavage of structural proteins during the assembly of the head of bacteriophage T4. Nature, London 227, 680-685.

Leak, D. J. \& Dalton, H. (1983). In vivo studies of primary alcohols, aldehydes and carboxylic acids as electron donors for the methane mono-oxygenase in a variety of methanotrophs. Journal of General Microbiology 129, 3487-3497.

Patel, R. N. (1984). Methane mono-oxygenase from Methylobacterium sp. strain CRL-26. In Microbial Growth on $C_{1}$ Compounds, Proceedings of the 4th International Symposium, pp. 83-90. Edited by R. L. Crawford \& R. S. Hanson. Washington, DC: American Society for Microbiology.

Peterson, G. L. (1977). A simplification of the protein assay of Lowry et al. which is more generally applicable. Analytical Biochemistry 83, 346-356. 
PrioR, S. D. \& Dalton, H. (1985). The effect of copper ions on membrane content and methane monooxygenase activity in methanol-grown cells of Methylococcus capsulatus (Bath). Journal of General Microbiology 131, 155-163.

Ribbons, D. W. \& WadzinsKi, A. M. (1976). Oxidation of $\mathrm{C}_{1}$ compounds by particulate fractions from Methylococcus capsulatus. In Symposium on Microbial Production and Utilisation of Gases $\left(\mathrm{H}_{2}, \mathrm{CH}_{4}, \mathrm{CO}\right)$, pp. 359-369. Edited by H. G. Schlegel, G. Gottschalk \& N. Pfennig. Göttingen: Akademie der Wissenschaften zu Göttingen

ScotT, D., Brannan, J. \& Higgins, I. J. (1981). The effect of growth conditions on intracytoplasmic membranes and methane mono-oxygenase activities in Methylosinus trichosporium OB3b. Journal of General Microbiology 125, 63-72.

Stanley, S. H., PrioR, S. D., Leak, D. J. \& Dalton, H. (1983). Copper stress underlies the fundamental change in intracellular location of methane monooxygenase in methane-oxidising organisms: studies in batch and continuous culture. Biotechnology Letters 5, 487-492.

Stirling, D. I., Colby, J. \& Dalton, H. (1979). A comparison of the methane mono-oxygenases from three strains of methane-oxidising bacteria. Biochemical Journal 177, 361-364.

Tonge, G. M., Harrison, D. E. F., KNowles, C. J. \& Higgins, I. J. (1975). Properties and partial purification of the methane-oxidising enzyme system from Methylosinus trichosporium. FEBS Letters 58, 293299.

Tonge, G. M., Harrison, D. E. F. \& Higgins, I. J. (1977). Purification and properties of the methane mono-oxygenase enzyme system from Methylosinus trichosporium OB3b. Biochemical Journal 161, 333344.

Woodland, M. P. \& Dalton, H. (1984). Purification and properties of component $\mathrm{A}$ of the methane mono-oxygenase from Methylococcus capsulatus (Bath). Journal of Biological Chemistry 259, 53-59. 